\title{
Adiponectin SNP45TG is associated with gestational diabetes mellitus
}

\begin{abstract}
INTRODUCTION: Diabetes and pregnancy can be associated in two ways: pregnancy that occurs in women who are already diabetic (diabetes of pre-gestational origin); and diabetes that occur in women who are already pregnant [gestational diabetes mellitus (GDM) (O'sullivan 1961)]. Patients with previous GDM history have higher risk of developing diabetes outside of pregnancy. Accumulating literature had suggested that adiponectin plays a role in the pathophysiology of this metabolic syndrome, and several of the common single nucleotide polymorphisms (SNP) in adiponectin gene have been identified in type 2 diabetes. Thus, one of the commonly found SNP was studied to determine its association with GDM. OBJECTIVE: To identify the association of SNP45TG with GDM. METHODS: This is a cross-sectional study involving pregnant mothers of <18 gestational weeks, who were recruited from three local antenatal clinics in Selangor, Malaysia. Their genomic DNA was extracted from EDTA treated whole blood using commercialized kit. Adiponectin gene was amplified through conventional PCR and SNP was detected using restriction enzyme SmaI. Plasma adiponectin level, fructosamine level and $\mathrm{HbA}(1 \mathrm{c})$ percentage were also examined. RESULTS: Among the 79 antenatal patients recruited, 53 patients were normal and 26 were diagnosed with GDM. Among the 53 normal patients, 18 carry TG/GG genotype. Meanwhile, among the 26 patients that were diagnosed with GDM 15 carry TG/GG genotype. Significant association was found between SNP45TG with GDM $(\chi(2)=4.038 ; \mathrm{P}<0.05)$. In addition, normal patients with TT genotype have significantly higher plasma adiponectin level compared to other groups. CONCLUSION: We concluded that SNP45TG in adiponectin gene is associated with the occurrence of GDM.
\end{abstract}

Keyword: Adiponectin;Single nucleotide polymorphism; SNP45; Gestational diabetes mellitus 